Journal of Science $\quad$ Gazi University

\title{
On Cauchy Numbers and Their Generalizations
}

\author{
Levent KARGIN \\ Akdeniz University, Faculty of Science Department of Mathematics, 07058 Antalya, Turkey
}

Highlights

- This paper focuses on a generalization of Cauchy numbers of both kinds.

- Some properties of this generalization are examined.

- Several new identities for classical Cauchy numbers of both kinds are obtained.

\section{Article Info}

Received: 09/08/2019 Accepted: $22 / 11 / 2019$

\section{Keywords}

Cauchy numbers Poly-Cauchy numbers p-Cauchy numbers Generating function Recurrence relations

\begin{abstract}
This paper is concerned with both kinds of the Cauchy numbers and their generalizations. Taking into account Mellin derivative, we relate $p$-Cauchy numbers of the second kind with shifted Cauchy numbers of the first kind, which yields new explicit formulas for the Cauchy numbers of the both kind. We introduce a generalization of the Cauchy numbers and investigate several properties, including recurrence relations, convolution identities and generating functions. In particular, these results give rise to new identities for Cauchy numbers.
\end{abstract}

\section{INTRODUCTION}

The Cauchy numbers of the first kind (CNFK) $c_{n}$ and the Cauchy numbers of the second kind (CNSK) $\hat{c}_{n}$ are given by the integral representations [1]

$$
c_{n}=\int_{0}^{1}(x)^{n} d x \text { and } \hat{c}_{n}=\int_{0}^{1}(x)^{\bar{n}} d x,
$$

where $(x)^{\underline{n}}=x(x-1) \cdots(x-n+1)$ with $(x)^{0}=1$ and $(x)^{\bar{n}}=x(x+1) \cdots(x+n-1)$ with $(x)^{\bar{n}}=$ 1. The numbers $c_{n}$, also known as Bernoulli numbers of the second kind $b_{n}$ with the relation $c_{n}=n ! b_{n}$ (see $[2,3]$ ), have the generating function

$$
\sum_{n=0}^{\infty} c_{n} \frac{z^{n}}{n !}=\frac{z}{\ln (1+z)}
$$

and satisfy the combinatorial formula

$$
c_{n}=\sum_{k=0}^{n}\left[\begin{array}{l}
n \\
k
\end{array}\right] \frac{(-1)^{n-k}}{k+1}
$$


Here $\left[\begin{array}{l}n \\ k\end{array}\right]$ are the (unsigned) Stirling numbers of the first kind, defined by

$$
\frac{\ln ^{k}(1+z)}{k !}=\sum_{n=k}^{\infty}\left[\begin{array}{l}
n \\
k
\end{array}\right](-1)^{n-k} \frac{z^{n}}{n !}
$$

The numbers $\hat{c}_{n}$ are generated by the function

$$
\sum_{n=0}^{\infty} \hat{c}_{n} \frac{z^{n}}{n !}=\frac{z}{(1+z) \ln (1+z)}
$$

and have an explicit formula

$$
\hat{c}_{n}=(-1)^{n} \sum_{k=0}^{n}\left[\begin{array}{l}
n \\
k
\end{array}\right] \frac{1}{k+1}
$$

It is good to note that the numbers $\hat{c}_{n}$ are also called as Nörlund numbers and denoted by $B_{n}^{(n)}$ [4].

Recently, many studies have been carried out on generalizations of Cauchy numbers, according to the generating functions [5, 6], integral representations [7, 8, 9] and explicit formulas [10,11, 12]. One of the generalizations of $c_{n}$ (resp. $\left.\hat{c}_{n}\right)$ is $c_{n}^{(q)}(a)$, called shifted poly-CNFK, (resp. $\hat{c}_{n}^{(q)}(a)$ shifted poly-CNSK) and defined by [13]

$$
\begin{gathered}
c_{n}^{(q)}(a)=\underbrace{\int_{0 \text { times }}^{1} \cdots \int_{0}^{1}\left(x_{1} \cdots x_{q}\right)^{a-1}\left(x_{1} \cdots x_{q}\right)^{\frac{n}{n}} d x_{1} \cdots d x_{q},}_{\hat{c}_{n}} \\
\hat{c}_{n}^{(q)}(a)=\underbrace{\int_{0}^{1} \cdots \int_{0}^{1}\left(x_{1} \cdots x_{q}\right)^{a-1}\left(x_{1} \cdots x_{q}\right)^{\bar{n}} d x_{1} \cdots d x_{q} .}_{\text {qtimes }}
\end{gathered}
$$

These numbers have the generating functions

$$
\sum_{n=0}^{\infty} c_{n}^{(q)}(a) \frac{z^{n}}{n !}=e_{q}(\ln (1+z), a)
$$

and

$$
\sum_{n=0}^{\infty} \hat{c}_{n}^{(q)}(a) \frac{z^{n}}{n !}=e_{q}(-\ln (1+z), a)
$$

where $e_{q}(z, a)$ is the poly-exponential function defined by [14]

$$
e_{q}(z, a)=\sum_{k=0}^{\infty} \frac{z^{k}}{k !(k+a)^{q}} .
$$

Moreover, these numbers can be written explicitly as [13] 


$$
c_{n}^{(q)}(a)=\sum_{k=0}^{n}\left[\begin{array}{l}
n \\
k
\end{array}\right] \frac{(-1)^{n-k}}{(k+a)^{q}} \text { and } \hat{c}_{n}^{(q)}(a)=(-1)^{n} \sum_{k=0}^{n}\left[\begin{array}{l}
n \\
k
\end{array}\right] \frac{1}{(k+a)^{q}} .
$$

If $q=1$ and $a=q=1$, we have shifted Cauchy and Cauchy numbers of the both kinds (CNBK), respectively.

Another generalization of Cauchy numbers is the $p$-Cauchy numbers. The $p$-CNFK $c_{n, p}$ and the $p$-CNSK $\hat{c}_{n, p}$ are defined by [15]

$$
\begin{gathered}
\frac{c_{n, p}}{p+1}=\int_{0}^{1}(1-x)^{p}(x)^{n} d x \\
\frac{\hat{c}_{n, p}}{p+1}=\int_{0}^{1}(1-x)^{p}(x)^{\bar{n}} d x
\end{gathered}
$$

and satisfy the explicit formulas

$$
c_{n, p}=\sum_{k=0}^{n}\left[\begin{array}{l}
n \\
k
\end{array}\right](-1)^{n-k}\left(\begin{array}{c}
k+p+1 \\
k
\end{array}\right)^{-1} \text { and } \hat{c}_{n, p}=(-1)^{n} \sum_{k=0}^{n}\left[\begin{array}{l}
n \\
k
\end{array}\right]\left(\begin{array}{c}
k+p+1 \\
k
\end{array}\right)^{-1}
$$

In particular, we have $c_{n, 0}=c_{n}$ and $\hat{c}_{n, 0}=\hat{c}_{n}$.

This study is composed of two main parts. In the first part, we obtain a relation between $p$-CNSK and shifted CNFK. This relation gives rise to some new results for the $p$-CNBK. In the second part, we introduce a further generalization of CNBK, including both shifted poly-CNBK and $p$-CNBK. So, we call these numbers shifted poly-p-Cauchy numbers of the both kinds. We then examine several properties of the shifted poly- $p$-CNBK, such as recurrence relations, convolution identities and generating functions. In particular, we reach new explicit formulas and recurrence relations for shifted poly-Cauchy, $p$-Cauchy and Cauchy numbers of the both kinds.

\section{2. $p$-CAUCHY NUMBERS}

In this section, we express $p$-Cauchy numbers in terms of Cauchy numbers. Moreover, we relate $p$-CNSK and shifted CNFK. Then, we obtain new explicit formulas for CNBK.

Komatsu [13] showed that

$$
c_{n}^{(q)}(a)=\sum_{\mu=0}^{a-1} \sum_{i=0}^{a-\mu-1}\left(\begin{array}{c}
a-1 \\
i
\end{array}\right)\left\{\begin{array}{c}
a-i-1 \\
\mu
\end{array}\right\} n^{i} c_{n+\mu}^{(q)}
$$

where $\left\{\begin{array}{l}n \\ k\end{array}\right\}$ is the Stirling numbers of the second kind, $a$ is a positive integer, $n$ is a non-zero integer and $0 \leq \mu \leq a-1$. In the following theorem, we give a different relation for shifted poly-CNFK.

Theorem 1. For all integers $n, p \geq 0, q \geq 1$ and positive real number $a$, we have

$$
c_{n}^{(q)}(a+p)=\sum_{k=0}^{p}\left\{\begin{array}{l}
p \\
k
\end{array}\right\} \sum_{l=0}^{k}\left(\begin{array}{l}
k \\
l
\end{array}\right)\left(\begin{array}{l}
n \\
l
\end{array}\right) l ! c_{n+k-l}^{(q)}(a) .
$$


Proof. Since

$$
\sum_{n=0}^{\infty}(x)^{n} \frac{z^{n}}{n !}=(1+z)^{x}
$$

(5) can be rewritten as

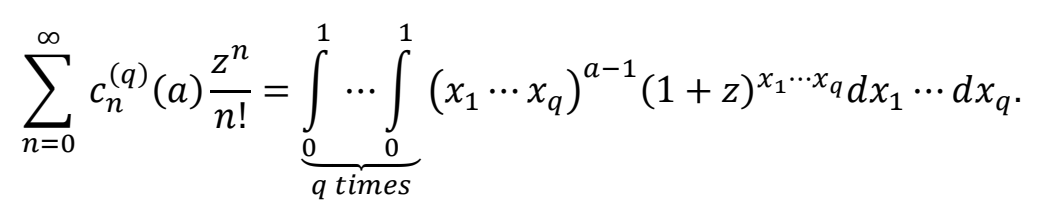

Applying the Mellin derivative, defined by $\left((1+z) \frac{d}{d z}\right)^{p}=\sum_{k=0}^{p}\left\{\begin{array}{l}p \\ k\end{array}\right\}(1+z)^{k} \frac{d^{k}}{d z^{k}}$ to the both sides of (11), we obtain

$$
\begin{aligned}
\sum_{n=0}^{\infty} c_{n}^{(q)}(a+p) & \frac{z^{n}}{n !} \\
= & \underbrace{1}_{0} \cdots \int_{0}^{1}\left(x_{1} \cdots x_{q}\right)^{a+p-1}(1+z)^{x_{1} \cdots x_{q}} d x_{1} \cdots d x_{q}=\left((1+z) \frac{d}{d z}\right)^{p} \sum_{n=0}^{\infty} c_{n}^{(q)}(a) \frac{z^{n}}{n !} \\
& =\sum_{n=0}^{\infty}\left[\sum_{k=0}^{p}\left\{\begin{array}{l}
p \\
k
\end{array}\right\} \sum_{l=0}^{k}\left(\begin{array}{l}
k \\
l
\end{array}\right)\left(\begin{array}{l}
n \\
l
\end{array}\right) l ! c_{n+k-l}^{(q)}(a)\right] \frac{z^{n}}{n !},
\end{aligned}
$$

which give the desired equation.

Setting $a=q=1$ in (9) and comparing with the following formula for $p$-CNSK [15]

$$
\frac{\hat{c}_{n-1, p+1}}{p+2}=\sum_{k=0}^{p}\left\{\begin{array}{l}
p \\
k
\end{array}\right\} \sum_{l=0}^{k}\left(\begin{array}{l}
k \\
l
\end{array}\right)\left(\begin{array}{l}
n \\
l
\end{array}\right) l ! c_{n+k-l}
$$

give the following relation.

Corollary 2. For $n \geq 1$ and $p \geq 0$,

$$
c_{n}(p+1)=\frac{1}{p+2} \hat{c}_{n-1, p+1}
$$

It is good to note that taking $p=0$ in (12) and using the relation [15]

$$
\hat{c}_{n+1, p}=\frac{p+1}{p+2} \hat{c}_{n, p+1}-(n+1) \hat{c}_{n, p}
$$

we have the well-known identity for the Cauchy numbers

$$
c_{n}=\hat{c}_{n}+n \hat{c}_{n-1}
$$

Moreover, from (12), we reach new explicit formulas as given in the following corollary.

Corollary 3. For $n, p \geq 0$, 


$$
\frac{1}{p+1} \hat{c}_{n, p}=\sum_{k=0}^{n}\left[\begin{array}{l}
n+1 \\
k+1
\end{array}\right] \frac{(-1)^{n-k}}{k+p+1}
$$

and

$$
c_{n+1}(p+1)=\frac{(-1)^{n}}{p+2} \sum_{k=0}^{n}\left[\begin{array}{l}
n \\
k
\end{array}\right]\left(\begin{array}{c}
k+p+2 \\
k
\end{array}\right)^{-1}
$$

When $p=0$, we have

$$
\hat{c}_{n}=\sum_{k=0}^{n}\left[\begin{array}{l}
n+1 \\
k+1
\end{array}\right] \frac{(-1)^{n-k}}{k+1}
$$

and

$$
c_{n+1}=(-1)^{n} \sum_{k=0}^{n}\left[\begin{array}{l}
n \\
k
\end{array}\right] \frac{1}{(k+1)(k+2)} .
$$

Now, we want to express $p$-CNFK in terms of CNFK. From (8), we have

$$
\frac{1}{p+1} \sum_{n=0}^{\infty} c_{n, p} \frac{z^{n}}{n !}=\int_{0}^{1}(1-x)^{p}(1+z)^{x} d x=\sum_{k=0}^{p}\left(\begin{array}{l}
p \\
k
\end{array}\right)(-1)^{k} \int_{0}^{1} x^{k}(1+z)^{x} d x
$$

Setting $q=1$ in (11) and using it in the above equation, we obtain

$$
\frac{1}{p+1} \sum_{n=0}^{\infty} c_{n, p} \frac{z^{n}}{n !}=\sum_{n=0}^{\infty}\left[\sum_{k=0}^{p}\left(\begin{array}{l}
p \\
k
\end{array}\right)(-1)^{k} c_{n}(k+1)\right] \frac{z^{n}}{n !}
$$

which can be written

$$
\frac{1}{p+1} c_{n, p}=\sum_{k=0}^{p}\left(\begin{array}{l}
p \\
k
\end{array}\right)(-1)^{k} c_{n}(k+1)
$$

Then, for $a=q=1$, using (9), we have the following theorem.

Theorem 4. For $n, p \geq 0$,

$$
\frac{1}{p+1} c_{n, p}=\sum_{i=0}^{p} \sum_{k=i}^{p} \sum_{l=0}^{i}\left(\begin{array}{l}
p \\
k
\end{array}\right)\left\{\begin{array}{l}
k \\
i
\end{array}\right\}\left(\begin{array}{l}
i \\
l
\end{array}\right)\left(\begin{array}{l}
n \\
l
\end{array}\right) l !(-1)^{k} c_{n+k-l} .
$$

Now, we want to recall the binomial transform which will be useful in the next theorem:

$$
a_{n}=\sum_{k=0}^{n}(-1)^{k}\left(\begin{array}{l}
n \\
k
\end{array}\right) b_{k}(n \geq 0) \text { if and only if } b_{n}=\sum_{k=0}^{n}(-1)^{k}\left(\begin{array}{l}
n \\
k
\end{array}\right) a_{k} .
$$

Using this and (18) give 


$$
c_{n}(p+1)=\sum_{k=0}^{p}\left(\begin{array}{l}
p \\
k
\end{array}\right) \frac{(-1)^{k} c_{n, k}}{k+1}
$$

From (12), the above equation can be written as

$$
\frac{1}{p+2} \hat{c}_{n, p+1}=\sum_{k=0}^{p}\left(\begin{array}{l}
p \\
k
\end{array}\right) \frac{(-1)^{k} c_{n+1, k}}{k+1}
$$

which is also a new recurrence relation between $p$-CNBK. For nonnegative integers $n$ and $p$, utilizing [15, Theorem 4.5]

$$
\frac{(-1)^{p}}{p+1} \mathrm{c}_{n, p}=\sum_{k=0}^{p}\left\{\begin{array}{l}
p \\
k
\end{array}\right\} \sum_{j=0}^{k+1}\left(\begin{array}{c}
k+1 \\
j
\end{array}\right)\left(\begin{array}{l}
n \\
j
\end{array}\right) j ! \hat{c}_{n-\mathrm{j}+\mathrm{k}}
$$

in the above equation, we have

$$
\frac{1}{p+2} \hat{c}_{n, p+1}=\sum_{k=0}^{p} \sum_{i=0}^{k} \sum_{l=0}^{i+1}\left(\begin{array}{l}
p \\
k
\end{array}\right)\left\{\begin{array}{l}
k \\
i
\end{array}\right\}\left(\begin{array}{c}
i+1 \\
l
\end{array}\right)\left(\begin{array}{c}
n+1 \\
l
\end{array}\right) l ! \hat{c}_{n+i+1-l} .
$$

Since

$$
\sum_{k=i}^{n}\left(\begin{array}{l}
n \\
k
\end{array}\right)\left\{\begin{array}{l}
k \\
i
\end{array}\right\}=\left\{\begin{array}{c}
n+1 \\
i+1
\end{array}\right\}
$$

we obtain a relation between $p$-CNSK and CNSK.

Theorem 5. For $n, p \geq 0$, we have

$$
\frac{1}{p+2} \hat{c}_{n, p+1}=\sum_{i=0}^{p}\left\{\begin{array}{c}
p+1 \\
i+1
\end{array}\right\} \sum_{l=0}^{i+1}\left(\begin{array}{c}
i+1 \\
l
\end{array}\right)\left(\begin{array}{c}
n+1 \\
l
\end{array}\right) l ! \hat{c}_{n+i+1-l}
$$

It is known that [13]

$$
(-1)^{n} c_{n}^{(q)}(a)=\sum_{m=1}^{n}\left[\begin{array}{l}
n \\
m
\end{array}\right\rfloor \hat{c}_{m}^{(q)}(a)
$$

where $\left[\begin{array}{l}n \\ m\end{array}\right\rfloor$ is the Lah numbers [16] defined by $\left[\begin{array}{l}n \\ m\end{array}\right\rfloor=\left(\begin{array}{l}n-1 \\ m-1\end{array}\right) \frac{n !}{m !}$. Setting $q=1$ and $a=p+1$ in (19) and using (12), we obtain a relation between $p$-CNSK and shifted CNSK.

Corollary 6. For $n, p \geq 0$, we have

$$
\frac{(-1)^{n+1}}{p+2} \hat{c}_{n, p+1}=\sum_{m=0}^{n}\left[\begin{array}{l}
n+1 \\
m+1
\end{array}\right\rfloor \hat{c}_{m+1}(p+1) .
$$




\section{SHIFTED POLY-p-CAUCHY NUMBERS OF THE FIRST KIND}

Let $n \geq 0, q \geq 1, p \geq 0$ be integers. Let $a$ be a positive real number. We define a sequence of rational numbers $c_{n, p}^{(q)}(a)$ by

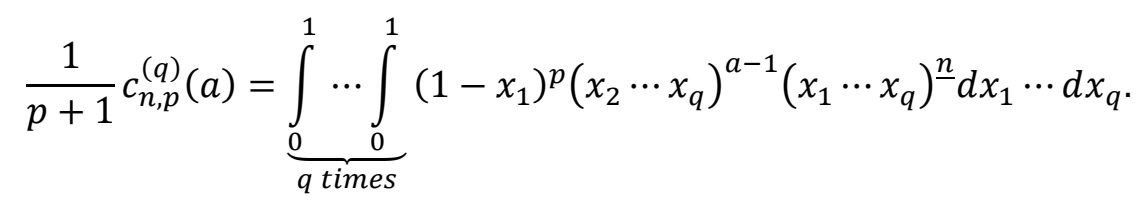

Since $(x)^{n}=\sum_{k=0}^{n}\left[\begin{array}{l}n \\ k\end{array}\right](-1)^{n-k} x^{k}$, we obtain the following explicit formula for $c_{n, p}^{(q)}(a)$.

Theorem 7. For all integers $n \geq 0, q \geq 1, p \geq-1$ and a positive real number $a$, we have

$$
c_{n, p}^{(q)}(a)=\sum_{k=0}^{n}\left[\begin{array}{l}
n \\
k
\end{array}\right]\left(\begin{array}{c}
k+p+1 \\
k
\end{array}\right)^{-1} \frac{(-1)^{n-k}}{(k+a)^{q-1}}
$$

From (21), it is evident that

$$
c_{n, p}^{(1)}(a)=c_{n, p}, c_{n,-1}^{(q)}(a)=c_{n}^{(q-1)}(a), c_{n, 0}^{(q)}(1)=c_{n}^{(q)}, c_{n, 0}^{(1)}(a)=c_{n,-1}^{(2)}(1)=c_{n},
$$

where $c_{n}^{(q)}$ is the $n$-th poly-CNFK [8]. Because of these relations we may call shifted poly-p-CNFK for $c_{n, p}^{(q)}(a)$.

Using Stirling transform, one can obtain that

$$
\sum_{k=0}^{n}\left\{\begin{array}{l}
n \\
k
\end{array}\right\} c_{k, p}^{(q)}(a)=\left(\begin{array}{c}
k+p+1 \\
k
\end{array}\right)^{-1} \frac{1}{(k+a)^{q-1}}
$$

We also note that such relations have been obtained for poly-Cauchy numbers in [8], for shifted polyCauchy numbers in [13] and for $p$-Cauchy numbers in [15]. Now, we want to generalize these relations with the use of generalized Stirling transform [17, Corollary 1]. We then obtain a relation between $r$-Stirling numbers of the second kind $\left\{\begin{array}{c}n+r \\ m+r\end{array}\right\}_{r}$ and shifted poly- $p$-CNFK.

Corollary 8. For $m \geq 0$, we have

$$
\sum_{k=0}^{n}\left\{\begin{array}{l}
n+m \\
k+m
\end{array}\right\}_{m} c_{m+k, p}^{(q)}(a)=\sum_{k=0}^{m}\left[\begin{array}{l}
m \\
k
\end{array}\right]\left(\begin{array}{c}
k+n+p+1 \\
k+n
\end{array}\right)^{-1} \frac{(-1)^{m-k}}{(k+n+a)^{q-1}}
$$

Using (10) and (20), the generating function of the number $c_{n, p}^{(q)}(a)$ satisfy the following iterated integrals.

Theorem 9. For all integers $q \geq 1, p \geq 0$ and a real number $a \geq 1$, we have

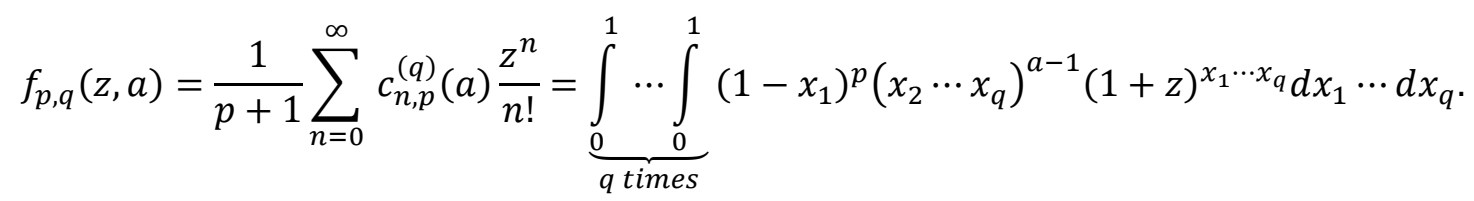

For integers $p \geq-1$ and $q$ and a positive real number $a$, define the function $e_{p, q}(z, a)$ by 


$$
e_{p, q}(z, a)=\sum_{k=0}^{\infty} \frac{z^{k}}{(p)^{\bar{k}}(k+a)^{q}}
$$

When $p=1$, we have $e_{1, q}(z, a)=e_{q}(z, a)$. Using (21), we have

$$
\sum_{n=0}^{\infty} c_{n, p}^{(q)}(a) \frac{z^{n}}{n !}=\sum_{k=0}^{\infty} \frac{k !}{(p+2)^{\bar{k}}(k+a)^{q-1}} \sum_{n=k}^{\infty}\left[\begin{array}{l}
n \\
k
\end{array}\right](-1)^{n-k} \frac{z^{n}}{n !}=\sum_{k=0}^{\infty} \frac{k !}{(p+2)^{\bar{k}}(k+a)^{q-1}} \frac{\ln ^{k}(1+z)}{k !}
$$

which can be stated as the following theorem.

Theorem 10. The number $c_{n, p}^{(q)}(a)$ have the following generating function

$$
\sum_{n=0}^{\infty} c_{n, p}^{(q)}(a) \frac{z^{n}}{n !}=e_{p+2, q-1}(\ln (1+z), a)
$$

The first few generating functions for $c_{n, p}^{(q)}(a)$ (for $\left.p=0,1,2\right)$ are

$$
\begin{aligned}
\sum_{n=0}^{\infty} c_{n, 0}^{(q)}(a) \frac{z^{n}}{n !}=\frac{-1}{(a-1)^{q-1} \ln (1+z)}+\frac{e_{q-1}(\ln (1+z), a-1)}{\ln (1+z)} & \\
\sum_{n=0}^{\infty} c_{n, 1}^{(q)}(a) \frac{z^{n}}{n !}= & \frac{-2}{(a-2)^{q-1} \ln ^{2}(1+z)}-\frac{2}{(a-1)^{q-1} \ln (1+z)}+\frac{2 e_{q-1}(\ln (1+z), a-2)}{\ln ^{2}(1+z)} \\
\sum_{n=0}^{\infty} c_{n, 2}^{(q)}(a) \frac{z^{n}}{n !} & =\frac{6}{(a-3)^{q-1} \ln ^{3}(1+z)}-\frac{3}{(a-2)^{q-1} \ln ^{2}(1+z)}-\frac{3}{(a-1)^{q-1} \ln (1+z)} \\
& +\frac{6 e_{q-1}\left(\ln ^{2}(1+z), a-3\right)}{\ln ^{3}(1+z)}
\end{aligned}
$$

In general, we state the following closed formula for the generating function of shifted poly- $p$-CNFK.

Theorem 11. For integers $q \geq 1, p \geq 0$ and a real number $a \geq p+2$,

$$
\sum_{n=0}^{\infty} c_{n, p}^{(q)}(a) \frac{z^{n}}{n !}=\frac{(p+1) !}{\ln ^{p+1}(1+z)} e_{q-1}(\ln (1+z), a-p-1)-\sum_{i=0}^{p} \frac{(p+1)^{i+1}}{(a-i-1)^{q-1} \ln ^{i+1}(1+z)}
$$

Proof. After an integration by parts, (22) can be written as

$$
f_{p, q}(z, a)=\frac{-1}{(a-1)^{q-1} \ln (1+z)}+\frac{p}{\ln (1+z)} f_{p-1, q}(z, a-1) .
$$

Applying inductively, we obtain

$$
f_{p, q}(z, a)=\frac{p !}{\ln ^{p}(1+z)} f_{0, q}(z, a-p)-\sum_{i=1}^{p} \frac{(p)^{\underline{i-1}}}{(a-i)^{q-1} \ln ^{i}(1+z)} .
$$

Setting $p=0$ and $a=a-p$ in (22), we have 


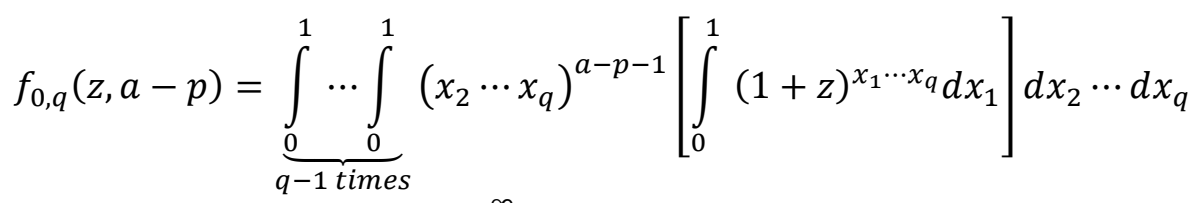

$$
\begin{aligned}
& =\frac{1}{\ln (1+z)}\left[\sum_{n=0}^{\infty} c_{n}^{(q-1)}(a-p-1) \frac{z^{n}}{n !}-\frac{1}{(a-p-1)^{q-1}}\right] .
\end{aligned}
$$

Using (6) in the above equation yields the formula.

It is good to note that setting $q=1$ in (24) gives [15]

$$
\sum_{n=0}^{\infty} c_{n, p} \frac{z^{n}}{n !}=\frac{(p+1) !(1+z)}{\ln ^{p+1}(1+z)}-\sum_{i=0}^{p} \frac{(p+1)^{i+1}}{\ln ^{i+1}(1+z)}
$$

Moreover, we have the following formula as an application of Theorem 11.

Theorem 12. For all integers $p \geq 0, n \geq p+1, q \geq 2$ and real numbers $a \geq p+2$, we have

$$
\begin{aligned}
\sum_{k=p+1}^{n}\left(\begin{array}{l}
n \\
k
\end{array}\right)\left[\begin{array}{c}
k \\
p+1
\end{array}\right](-1)^{k+p+1} c_{n-k, p}^{(q)}(a) & \\
& =c_{n}^{(q-1)}(a-p-1)+(-1)^{n+1} \sum_{i=0}^{p}\left[\begin{array}{l}
n \\
i
\end{array}\right] \frac{(-1)^{i}}{(a+i-p-1)^{q-1}} .
\end{aligned}
$$

For $q=1$, we have

$$
\sum_{k=p+1}^{n}\left(\begin{array}{l}
n \\
k
\end{array}\right)\left[\begin{array}{c}
k \\
p+1
\end{array}\right](-1)^{k+p+1} c_{n-k, p}=(-1)^{n+1} \sum_{i=0}^{p}\left[\begin{array}{l}
n \\
i
\end{array}\right](-1)^{i}, \quad n \geq 2
$$

We remark that for $p=0$ in (29), using the relation $c_{n}=n ! b_{n}$ and the well-known identity $\left[\begin{array}{l}n \\ 1\end{array}\right]=(n-1)$ !, we have

$$
\sum_{k=1}^{n}(-1)^{k+1}\left(\begin{array}{l}
n \\
k
\end{array}\right)(k-1) ! c_{n-k}=0, \quad n \geq 2 .
$$

which was proved by Agoh and Dilcher [2] with a different method.

Proof of Theorem 12. Multiply both sides of (24) by $\frac{\ln ^{p+1}(1+z)}{(p+1) !}$ and use (3) to obtain (28). Moreover, multiply both sides of (27) by $\frac{\ln ^{p+1}(1+z)}{(p+1) !}$ and use (3) to have (29).

Now, we want to deal with some recurrence relations of shifted poly- $p$-CNFK. We first have the following:

Theorem 13. For all integers $n \geq 0, q \geq 1$ and real numbers $a \geq 1$, we have

$$
c_{n+1, p}^{(q)}(a)+n c_{n, p}^{(q)}(a)=\frac{-(p+1)}{p+2} c_{n, p+1}^{(q)}(a+1)+c_{n, p}^{(q)}(a+1), \quad p \geq-1
$$


and

$$
(a-p-1) c_{n, p}^{(q+1)}(a)=c_{n, p}^{(q)}(a)-(p+1) c_{n, p-1}^{(q+1)}(a), \quad p \geq 0
$$

Proof. Differentiate both sides of (22) with respect to $z$ and multiply it by $(1+z)$ to obtain

$$
\begin{aligned}
(1+z) \frac{d}{d z} f_{p, q}(z, a) & =\underbrace{1}_{\underbrace{}_{0} \cdots \int_{0}^{1}}\left(1-x_{1}\right)^{p} x_{1}\left(x_{2} \cdots x_{q}\right)^{a}(1+z)^{x_{1} \cdots x_{q}} d x_{1} \cdots d x_{q} \\
& =-\int_{0 \text { times }}^{\int_{0}^{1} \cdots \int_{0}^{1}}\left(1-x_{1}\right)^{p+1}\left(x_{2} \cdots x_{q}\right)^{a}(1+z)^{x_{1} \cdots x_{q}} d x_{1} \cdots d x_{q} \\
& +\underbrace{\int_{0}^{1} \cdots \int_{0}^{1}\left(1-x_{1}\right)^{p}\left(x_{2} \cdots x_{q}\right)^{a}(1+z)^{x_{1} \cdots x_{q}} d x_{1} \cdots d x_{q},}_{q \text { times }}
\end{aligned}
$$

or, equivalently,

$$
\frac{(1+z)}{p+1} \sum_{n=0}^{\infty} c_{n+1, p}^{(q)}(a) \frac{z^{n}}{n !}=\frac{-1}{p+2} \sum_{n=0}^{\infty} c_{n, p+1}^{(q)}(a+1) \frac{z^{n}}{n !}+\frac{1}{p+1} \sum_{n=0}^{\infty} c_{n, p}^{(q)}(a+1) \frac{z^{n}}{n !} .
$$

After some rearrangement, we obtain (31).

One can obtain that

$$
e_{p, q}(x, a)=\frac{1}{a^{q}}{ }_{p} F_{q+1}\left(\begin{array}{l}
1, a, \ldots, a \\
p, a+1, \ldots a+1
\end{array} ; x\right)
$$

where ${ }_{p} F_{q}\left(\begin{array}{l}a_{1}, \ldots, a_{p} \\ b_{1}, \ldots b_{q}\end{array} ; x\right)$ is the generalized hypergeometric function defined by

$$
{ }_{p} F_{q}\left(\begin{array}{l}
a_{1}, \ldots, a_{p} \\
b_{1}, \ldots b_{q}
\end{array} ; x\right)=\sum_{k=0}^{\infty} \frac{\left(a_{1}\right)^{\bar{k}} \cdots\left(a_{p}\right)^{\bar{k}}}{\left(b_{1}\right)^{\bar{k}} \cdots\left(b_{q}\right)^{\bar{k}}} \frac{x^{k}}{k !}
$$

For $p=p+2$ and $x=\ln (1+z)$, we have

$$
\begin{aligned}
& (a-p-1)_{q+1} F_{q+1}\left(\begin{array}{l}
1, a, \ldots, a \\
p, a+1, \ldots a+1
\end{array} ; \ln (1+z)\right) \\
& =a_{q+1} F_{q+1}\left(\begin{array}{l}
1, a+1, a \ldots, a \\
p, a+1, \ldots a+1
\end{array} ; \ln (1+z)\right) \\
& -(p-1)_{q+1} F_{q+1}\left(\begin{array}{l}
1, a \ldots, a \\
p-1, a+1, \ldots a+1
\end{array} ; \ln (1+z)\right) \text {. }
\end{aligned}
$$

From (23) and (33), we obtain 


$$
(a-p-1) \sum_{n=0}^{\infty} c_{n, p}^{(q+1)}(a) \frac{z^{n}}{n !}=\sum_{n=0}^{\infty} c_{n, p}^{(q)}(a) \frac{z^{n}}{n !}-(p+1) \sum_{n=0}^{\infty} c_{n, p-1}^{(q+1)}(a) \frac{z^{n}}{n !}
$$

which gives (32).

Secondly, we obtain the following recurrence relations which are generalizations of (30).

Theorem 14. For all integers $n \geq 1, q \geq 1, p \geq 0$ and real number $a>1$ we have

$$
\sum_{k=1}^{n}(-1)^{k+1}\left(\begin{array}{l}
n \\
k
\end{array}\right)(k-1) ! c_{n-k, p}^{(q)}(a)=(p+1) c_{n, p-1}^{(q)}(a-1)
$$

In particular,

$$
\begin{gathered}
\sum_{k=1}^{n}(-1)^{k+1}\left(\begin{array}{l}
n \\
k
\end{array}\right)(k-1) ! c_{n-k}^{(q)}(a)=c_{n}^{(q-1)}(a-1)-(a-1) c_{n}^{(q)}(a-1), q \geq 2 \\
\sum_{k=1}^{n}(-1)^{k+1}\left(\begin{array}{l}
n \\
k
\end{array}\right)(k-1) ! c_{n-k, p}=(p+1) c_{n, p-1}, p \geq 1
\end{gathered}
$$

Proof. Considering (23) and (25) yield

$$
\frac{\ln (1+z)}{p+1} \sum_{n=0}^{\infty} c_{n, p}^{(q)}(a) \frac{z^{n}}{n !}=\sum_{n=1}^{\infty} c_{n, p-1}^{(q)}(a-1) \frac{z^{n}}{n !}
$$

Then, we have

$$
\begin{array}{r}
(p+1) \sum_{n=1}^{\infty} c_{n, p-1}^{(q)}(a-1) \frac{z^{n}}{n !}=\sum_{k=0}^{\infty} \sum_{n=0}^{\infty} \frac{(-1)^{k} c_{n, p}^{(q)}(a)}{(k+1) n !} z^{n+k+1} \\
=\sum_{n=1}^{\infty} \sum_{k=1}^{n}(-1)^{k+1}\left(\begin{array}{l}
n \\
k
\end{array}\right)(k-1) ! c_{n-k, p}^{(q)}(a) \frac{z^{n}}{n !}
\end{array}
$$

Comparing the coefficients of $\frac{z^{n}}{n !}$ in the both sides of the above equation gives (34).

From (32), (34) can be written as

$$
\sum_{k=1}^{n}(-1)^{k+1}\left(\begin{array}{l}
n \\
k
\end{array}\right)(k-1) ! c_{n-k, p}^{(q)}(a)=c_{n, p}^{(q-1)}(a-1)-(a-p-2) c_{n, p}^{(q)}(a-1) .
$$

Setting $p=-1$ in the above equation yields (35).

Komatsu [18] showed that

$$
\sum_{k=0}^{n}\left(\begin{array}{l}
n \\
k
\end{array}\right) c_{k} c_{n-k}^{(q)}=c_{n}+n c_{n-1}-n \sum_{j=1}^{q}\left(c_{n}^{(j)}+(n-1) c_{n-1}^{(j)}\right),
$$

as a generalization of [19] 


$$
\sum_{k=0}^{n}\left(\begin{array}{l}
n \\
k
\end{array}\right) c_{k} c_{n-k}=-(n-1) c_{n}-n(n-2) c_{n-1} .
$$

We give analogues of those results in the following theorem.

Theorem 15. For all integers $n \geq 1, q \geq 1, p \geq 0$ and real number $a>1$, we have

$$
\sum_{k=0}^{n}\left(\begin{array}{l}
n \\
k
\end{array}\right) c_{k} c_{n-k, p-1}^{(q)}(a-1)=\frac{c_{n}}{(a-1)^{q-1}}+\frac{n c_{n-1, p}^{(q)}(a)}{p+1}
$$

When $q=1$, it becomes

$$
\sum_{k=0}^{n}\left(\begin{array}{l}
n \\
k
\end{array}\right) c_{k} c_{n-k, p-1}=c_{n}+\frac{n}{p+1} c_{n-1, p}
$$

It is good to note that for $a=2$ and $p=0$ in (38), we have

$$
\sum_{k=0}^{n}\left(\begin{array}{l}
n \\
k
\end{array}\right) c_{k} c_{n-k}^{(q-1)}=n c_{n-1,0}^{(q)}(2)+c_{n}
$$

Comparing (37) with the above equation, we conclude that

$$
\sum_{j=1}^{q-1}\left(c_{n}^{(j)}+(n-1) c_{n-1}^{(j)}\right)=c_{n-1}-c_{n-1,0}^{(q)}(2)
$$

On the other hand, setting $p=-1$ and $a=1$ in (31), we obtain $c_{n+1}^{(q-1)}+n c_{n}^{(q-1)}=c_{n}^{(q-1)}(2)$. Using this and (39), we have another conclusion as

$$
\sum_{j=1}^{q-1} c_{n}^{(j)}(2)=c_{n}-c_{n, 0}^{(q)}(2)
$$

Proof of Theorem 15. With use of (1), (23) and (25), we have

$$
\begin{gathered}
\frac{1}{p+1} \sum_{n=0}^{\infty} c_{n, p}^{(q)}(a) \frac{z^{n+1}}{n !}+\frac{1}{(a-1)^{q-1}} \sum_{n=0}^{\infty} c_{n} \frac{z^{n}}{n !}=\sum_{k=0}^{\infty} \sum_{n=0}^{\infty} \frac{c_{k} c_{n, p-1}^{(q)}(a-1)}{k ! n !} z^{n+k} \\
=\sum_{n=0}^{\infty}\left[\sum_{k=0}^{n}\left(\begin{array}{l}
n \\
k
\end{array}\right) c_{k} c_{n-k, p-1}^{(q)}(a-1)\right] \frac{z^{n}}{n !} .
\end{gathered}
$$

Comparing the coefficients of $\frac{z^{n}}{n !}$ in the both sides of the above equation completes the proof.

\section{SHIFTED POLY-p-CAUCHY NUMBERS OF THE SECOND KIND}

Let $a$ be a positive real number. We define shifted poly- $p$-CNSK $\hat{c}_{n, p}^{(q)}(a)(n \geq 0, q \geq 1, p \geq 0)$ by 


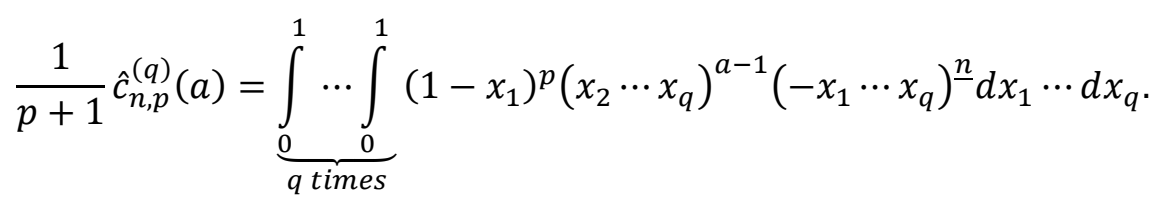

Then, similar to Theorem $7, \hat{c}_{n, p}^{(q)}(a)$ have the following explicit formula.

Theorem 16. For all integers $n \geq 0, q \geq 1, p \geq-1$ and positive real number $a$,

$$
\hat{c}_{n, p}^{(q)}(a)=(-1)^{n} \sum_{k=0}^{n}\left[\begin{array}{l}
n \\
k
\end{array}\right]\left(\begin{array}{c}
k+p+1 \\
k
\end{array}\right)^{-1} \frac{1}{(k+a)^{q-1}} .
$$

In particular, we have

$$
\hat{c}_{n, p}^{(1)}(a)=\hat{c}_{n, p}, \hat{c}_{n,-1}^{(q)}(a)=\hat{c}_{n}^{(q-1)}(a), \hat{c}_{n, 0}^{(q)}(1)=\hat{c}_{n}^{(q)}, \hat{c}_{n, 0}^{(1)}(a)=\hat{c}_{n,-1}^{(2)}(1)=\hat{c}_{n}
$$

where $\hat{c}_{n}^{(q)}$ is the n-th poly-CNSK.

The numbers $\hat{c}_{n, p}^{(q)}(a)$ have the following generating functions. The proof is similar to that of Theorem 9 and Theorem 10 and omitted.

Theorem 17. For all integers $q \geq 1, p \geq 0$ and positive real number $a$,

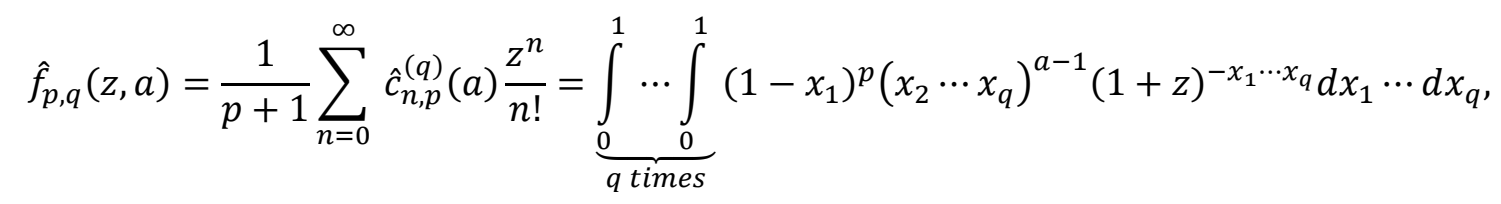

and

$$
\sum_{n=0}^{\infty} \hat{c}_{n, p}^{(q)}(a) \frac{z^{n}}{n !}=e_{p+2, q-1}(-\ln (1+z), a)=\frac{1}{a^{q-1}} q_{q} F_{q}\left(\begin{array}{l}
1, a, \ldots, a \\
p+2, a+1, \ldots, a+1
\end{array} ;-\ln (1+z)\right) .
$$

The first few generating function for $\hat{c}_{n, p}^{(q)}(a)$ (for $p=0,1$ ) are

$$
\begin{gathered}
\sum_{n=0}^{\infty} \hat{c}_{n, 0}^{(q)}(a) \frac{z^{n}}{n !}=\frac{1}{(a-1)^{q-1} \ln (1+z)}-\frac{e_{q-1}(-\ln (1+z), a-1)}{\ln (1+z)}, \\
\sum_{n=0}^{\infty} c_{n, 1}^{(q)}(a) \frac{z^{n}}{n !}=\frac{-2}{(a-2)^{q-1} \ln ^{2}(1+z)}+\frac{2}{(a-1)^{q-1} \ln (1+z)}+\frac{2 e_{q-1}(-\ln (1+z), a-2)}{\ln ^{2}(1+z)} .
\end{gathered}
$$

In general, we have the following closed formula for the generating function of $\hat{c}_{n, p}^{(q)}(a)$.

Theorem 18. For integers $q \geq 2, p \geq 0$ and a real number $a \geq p+2$, 


$$
\begin{aligned}
\sum_{n=0}^{\infty} \hat{c}_{n, p}^{(q)}(a) \frac{z^{n}}{n !} & =\frac{(-1)^{p+1}(p+1) !}{\ln ^{p+1}(1+z)} e_{q-1}(-\ln (1+z), a-p-1) \\
& +\sum_{i=0}^{p} \frac{(-1)^{i}(p+1) \frac{i+1}{(a-i-1)^{q-1} \ln ^{i+1}(1+z)}}{(}
\end{aligned}
$$

Proof. After an integration by parts, (41) can be written as

$$
\hat{f}_{p, q}(z, a)=\frac{1}{(a-1)^{q-1} \ln (1+z)}-\frac{p}{\ln (1+z)} \hat{f}_{p-1, q}(z, a-1) .
$$

Applying inductively, we obtain

$$
\hat{f}_{p, q}(z, a)=\frac{(-1)^{p} p !}{\ln ^{p}(1+z)} \hat{f}_{0, q}(z, a-p)+\sum_{i=1}^{p} \frac{(-1)^{i+1}(p) \frac{i-1}{(a-i)^{q-1} \ln ^{i}(1+z)}}{.}
$$

Setting $p=0, a=a-p$ in (41) and using (7), we have

$$
f_{0, q}(z, a-p)=\frac{1}{\ln (1+z)}\left[-e_{q-1}(-\ln (1+z), a-p-1)+\frac{1}{(a-p-1)^{q-1}}\right] .
$$

Utilizing the above equation in (44) completes the proof.

We note that for $q=1$ in (42), we have [15]

$$
\sum_{n=0}^{\infty} \hat{c}_{n, p} \frac{z^{n}}{n !}=\frac{(-1)^{p+1}(p+1) !}{(1+z) \ln ^{p+1}(1+z)}+\sum_{i=0}^{p} \frac{(-1)^{i}(p+1) \underline{i+1}}{\ln ^{i+1}(1+z)}
$$

Multiplying both sides of (42) with $\frac{\ln ^{p+1}(1+z)}{(p+1) !}$ and then applying the method used in the proof of Theorem 12, we arrive at the following:

Theorem 19. For integers $q \geq 2, n \geq p+1, p \geq 0$ and a real number $a \geq p+2$,

$$
\sum_{k=p+1}^{n}\left(\begin{array}{l}
n \\
k
\end{array}\right)\left[\begin{array}{c}
k \\
p+1
\end{array}\right](-1)^{k} \hat{c}_{n-k, p}^{(q)}(a)=\hat{c}_{n}^{(q-1)}(a-p-1)+(-1)^{n+1} \sum_{i=0}^{p}\left[\begin{array}{l}
n \\
i
\end{array}\right] \frac{1}{(a+i-p-1)^{q-1}}
$$

For $q=1$, we have

$$
\sum_{k=p+1}^{n}\left(\begin{array}{l}
n \\
k
\end{array}\right)\left[\begin{array}{c}
k \\
p+1
\end{array}\right](-1)^{k} \hat{c}_{n-k, p}=(-1)^{n} n !+(-1)^{n+1} \sum_{i=0}^{p}\left[\begin{array}{l}
n \\
i
\end{array}\right], \quad n \geq 1
$$

Remark that for $p=0$, (47) becomes

$$
\sum_{j=0}^{n-1} \frac{(-1)^{j}}{n-j} \frac{B_{j}^{(j)}}{j !}=1, \quad n \geq 1
$$

proved by Nörlund [4]. Moreover, using (12) in (47) yield ( $n \geq p+1, p \geq 1)$ 


$$
\sum_{k=p+1}^{n}\left(\begin{array}{l}
n \\
k
\end{array}\right)\left[\begin{array}{c}
k \\
p+1
\end{array}\right](-1)^{k} c_{n-k+1}(p)=\frac{(-1)^{n} n !}{p+1}+\frac{(-1)^{n+1}}{p+1} \sum_{i=0}^{p}\left[\begin{array}{l}
n \\
i
\end{array}\right]
$$

Setting $p=1$ in (49) and using the well-known identity

$$
\left[\begin{array}{l}
n \\
2
\end{array}\right]=(n-1) ! H_{n-1}
$$

where $H_{n}=1+\frac{1}{2}+\cdots+\frac{1}{n}$ is the harmonic number, we deduce an analogue of

$$
\sum_{k=0}^{n-2} \frac{(-1)^{k}}{k !(n-k)} H_{n-k-1} c_{k}=\frac{1}{2(n-1)}
$$

recorded by Agoh and Dilcher [2].

Corollary 20. For $n \geq 2$,

$$
\sum_{k=0}^{n-2} \frac{(-1)^{k}}{k !(n-k)} H_{n-k-1} c_{k+1}=\frac{(n-1)}{2 n}
$$

The following theorem relates both kinds of the shifted poly-p-Cauchy numbers.

Theorem 21. Let $q$ be an integer and a be a positive real number. For $n \geq 1$ and $p \geq-1$, we have

$$
(-1)^{n} \hat{c}_{n, p}^{(q)}(a)=\sum_{k=1}^{n}\left\lfloor\begin{array}{l}
n \\
k
\end{array}\right\rfloor c_{k, p}^{(q)}(a)
$$

and

$$
(-1)^{n} c_{n, p}^{(q)}(a)=\sum_{k=1}^{n}\left\lfloor\begin{array}{l}
n \\
k
\end{array}\right\rfloor \hat{c}_{k, p}^{(q)}(a)
$$

Proof. With the use of $(-x)^{\underline{n}}=(-1)^{n}(x)^{\bar{n}}$ and $(x)^{\bar{n}}=\sum_{k=1}^{n}\left|\begin{array}{l}n \\ k\end{array}\right|(x)^{\underline{k}}$ in (40), we have

$$
\frac{(-1)^{n}}{p+1} \hat{c}_{n, p}^{(q)}(a)=\sum_{k=1}^{n}\left|\begin{array}{l}
n \\
k
\end{array}\right| \underbrace{\int_{0}^{1} \cdots \int_{0}^{1}}_{q \text { times }}\left(1-x_{1}\right)^{p}\left(x_{2} \cdots x_{q}\right)^{a-1}\left(x_{1} \cdots x_{q}\right)^{\underline{k}} d x_{1} \cdots d x_{q}
$$

Then, from (20), we have the first identity. Moreover, let $\left(a_{n}\right)_{n=0}^{\infty}$ and $\left(b_{n}\right)_{n=0}^{\infty}$ be sequences of complex numbers. Then $b_{n}=\sum_{k=1}^{n}\left[\begin{array}{l}n \\ k\end{array}\right] a_{k}$ if and only if $a_{n}=\sum_{k=1}^{n}\left[\begin{array}{l}n \\ k\end{array}\right](-1)^{n-k} b_{k}$. Using this relation in the first identity yields the second formula.

These relations enable us to examine some properties of $\hat{c}_{n, p}^{(q)}(a)$ from $c_{n, p}^{(q)}(a)$. For instance, the following recurrence relations holds for shifted poly- $p$-CNSK.

Corollary 22. For all integers $n \geq 0, q \geq 1$ and real numbers $a \geq 1$, we have 


$$
\hat{c}_{n+1, p}^{(q)}(a)+n \hat{c}_{n, p}^{(q)}(a)=\frac{(p+1)}{p+2} \hat{c}_{n, p+1}^{(q)}(a+1)+\hat{c}_{n, p}^{(q)}(a+1), \quad p \geq-1
$$

and

$$
(a-p-1) \hat{c}_{n, p}^{(q+1)}(a)=\hat{c}_{n, p}^{(q)}(a)-(p+1) \hat{c}_{n, p-1}^{(q+1)}(a), \quad p \geq 0
$$

Proof. Multiply both sides of (31) by $\left[\begin{array}{l}m \\ n\end{array}\right\rfloor$ and sum over $n$ from 1 to $m$ to obtain

$$
\sum_{n=1}^{m}\left\lfloor\begin{array}{l}
m \\
n
\end{array}\right\rfloor c_{n+1, p}^{(q)}(a)+\sum_{n=1}^{m}\left\lfloor\begin{array}{l}
m \\
n
\end{array}\right\rfloor n c_{n, p}^{(q)}(a)=\frac{-(p+1)}{p+2} \sum_{n=1}^{m}\left\lfloor\begin{array}{l}
m \\
n
\end{array}\right\rfloor c_{n, p+1}^{(q)}(a+1)+\sum_{n=1}^{m}\left\lfloor\begin{array}{l}
m \\
n
\end{array}\right\rfloor c_{n, p}^{(q)}(a+1) .
$$

Using (51) and the recurrence relation of Lah numbers

$$
\left[\begin{array}{c}
m+1 \\
n
\end{array}\right\rfloor=\left\lfloor\begin{array}{c}
m \\
n-1
\end{array}\right\rfloor+(m+n)\left\lfloor\begin{array}{c}
m \\
n
\end{array}\right\rfloor
$$

we obtain (53). Similarly, using (32) and (51) gives (54).

Using (41) and (43) and applying the same method used for the proof of Theorem 14, we obtain the following recurrence relations which are the analogues of (48).

Theorem 23. For all integers $n \geq 1$, and real number $a>1$, we have

$$
\sum_{k=1}^{n}(-1)^{k}\left(\begin{array}{l}
n \\
k
\end{array}\right)(k-1) ! \hat{c}_{n-k, p}^{(q)}(a)=(p+1) \hat{c}_{n, p-1}^{(q)}(a-1), \quad q \geq 1, p \geq 0 .
$$

In particular,

$$
\begin{gathered}
\sum_{k=1}^{n}(-1)^{k}\left(\begin{array}{l}
n \\
k
\end{array}\right)(k-1) ! \hat{c}_{n-k}^{(q)}(a)=\hat{c}_{n}^{(q-1)}(a-1)-(a-1) \hat{c}_{n}^{(q)}(a-1), \quad q \geq 2, \\
\sum_{k=1}^{n}(-1)^{k}\left(\begin{array}{l}
n \\
k
\end{array}\right)(k-1) ! \hat{c}_{n-k, p}=(p+1) \hat{c}_{n, p-1}, \quad p \geq 1 .
\end{gathered}
$$

On the other hand, using (12) and (57), we have

$$
\sum_{k=1}^{n}(-1)^{k}\left(\begin{array}{l}
n \\
k
\end{array}\right)(k-1) ! c_{n+1-k}(p)=\hat{c}_{n, p-1}
$$

Setting $p=1$ in the above equation gives a new relation between CNBK.

Corollary 24. For $n \geq 1$, we have

$$
\sum_{k=1}^{n}(-1)^{k}\left(\begin{array}{l}
n \\
k
\end{array}\right)(k-1) ! c_{n+1-k}=\hat{c}_{n}
$$

Note that Howard [20] gave the relation 


$$
B_{n}^{(n)}=n ! \sum_{k=0}^{n}(-1)^{n-k} b_{k}
$$

Using the relation $c_{n}=n ! b_{n}$, one can easily see that (58) can be written in the following form

$$
B_{n}^{(n)}=n ! \sum_{k=1}^{n} \frac{(-1)^{n-k-1} k}{n+1-k} b_{k}
$$

Komatsu [18] gave

$$
\sum_{k=0}^{n}\left(\begin{array}{l}
n \\
k
\end{array}\right) c_{k} \hat{c}_{n-k}^{(q-1)}=\hat{c}_{n}-n \sum_{j=1}^{q-1}\left(\hat{c}_{n}^{(j)}+(n-1) \hat{c}_{n-1}^{(j)}\right)
$$

to generalize [19]

$$
\sum_{k=0}^{n}\left(\begin{array}{l}
n \\
k
\end{array}\right) c_{k} \hat{c}_{n-k}=-(n-1)\left(\hat{c}_{n}+n \hat{c}_{n-1}\right) .
$$

Now, we want to generalize these formulas. The proof is similar to that of Theorem 15 with the use of (41) and (43), so it is omitted.

Theorem 25. For all integers $n \geq 1, q \geq 1, p \geq 0$ and real number $a>1$, we have

$$
\sum_{k=0}^{n}\left(\begin{array}{l}
n \\
k
\end{array}\right) c_{k} \hat{c}_{n-k, p-1}^{(q)}(a-1)=\frac{c_{n}}{(a-1)^{q-1}}-\frac{n \hat{c}_{n-1, p}^{(q)}(a)}{p+1}
$$

When $q=1$, we obtain

$$
\sum_{k=0}^{n}\left(\begin{array}{l}
n \\
k
\end{array}\right) c_{k} \hat{c}_{n-k, p-1}=c_{n}-\frac{n}{p+1} \hat{c}_{n-1, p}
$$

It is good to note that for $a=2$ and $p=0$ in (60), we have

$$
\sum_{k=0}^{n}\left(\begin{array}{l}
n \\
k
\end{array}\right) c_{k} \hat{c}_{n-k}^{(q-1)}=c_{n}-n \hat{c}_{n-1,0}^{(q)}(2) .
$$

Comparing (59) with the above equation and using (13), we conclude that

$$
\sum_{j=1}^{q-1}\left(\hat{c}_{n}^{(j)}+(n-1) \hat{c}_{n-1}^{(j)}\right)=\hat{c}_{n-1,0}^{(q)}(2)-\hat{c}_{n-1} .
$$

On the other hand, setting $p=-1$ and $a=1$ in (53) and using it in the above equation, we arrive at

$$
\sum_{j=1}^{q-1} \hat{c}_{n}^{(j)}(2)=\hat{c}_{n, 0}^{(q)}(2)-\hat{c}_{n}
$$


Moreover, utilizing (12), (61) can be written as

$$
\sum_{k=0}^{n}\left(\begin{array}{l}
n \\
k
\end{array}\right) c_{k} c_{n+1-k}(p-1)=\frac{c_{n}}{p}-\frac{n c_{n}(p)}{p} .
$$

From (9), we have $c_{n}(2)=c_{n+1}+n c_{n}$. Thus, we obtain a new convolution identity for CNFK.

Corollary 26. For $n \geq 0$

$$
\sum_{k=0}^{n}\left(\begin{array}{l}
n \\
k
\end{array}\right) c_{k} c_{n+1-k}=\frac{\left(1-n^{2}\right) c_{n}-n c_{n+1}}{2}
$$

\section{CONCLUSUIONS}

In this paper, a new generalization of Cauchy numbers is introduced, and some arithmetical properties are discussed. In particular, new identities for Cauchy numbers are achieved. In the recent paper [21], HurwitzLerch type multi-poly-Cauchy numbers are studied. For the further research, considering this paper with our results, one can introduce the concept of Hurwitz-Lerch type multi-poly- $p$-Cauchy numbers.

\section{ACKNOWLEDGEMENTS}

We would like to thank the referees for their helpful comments on the manuscript. This work was supported by Research Fund of Akdeniz University. Project Number: FBA-2018-3723.

\section{CONFLICTS OF INTEREST}

No conflict of interest was declared by the author.

\section{REFERENCES}

[1] Comtet, L., Advanced Combinatorics, Reidel, Dordrecht, (1974).

[2] Agoh, T. and Dilcher, K., "Recurrence relations for Nörlund numbers and Bernoulli numbers of the second kind", Fibonacci Q., 48: 4-12, (2010).

[3] Young, P.T., "A 2-adic formula for Bernoulli numbers of the second kind and for the Nörlund numbers", J. Number Theory, 128: 2951-2962, (2008).

[4] Nörlund, N.E., Vorlesungen Äuber Direrenzenrechnung, Springer-Verlag, Berlin, (1924).

[5] Cenkci, M. and Young, P.T., "Generalizations of poly-Bernoulli and poly-Cauchy numbers", Eur. J. Math., 1:799-828, (2015).

[6] Komatsu, T., "Hypergeometric Cauchy numbers”, Int. J. Number Theory, 9: 545-560, (2013).

[7] Komatsu, T., Laohakosol,V., and Liptai, K., "A generalization of poly-Cauchy numbers and their properties”, Abstr. Appl. Anal., 2013: Article ID 179841, (2013).

[8] Komatsu, T., "Poly-Cauchy numbers”, Kyushu J. Math., 67: 143-153, (2013). 
[9] Komatsu, T., "Poly-Cauchy numbers with a q parameter", Raman. J., 31: 353-371, (2013).

[10] Komatsu, T., "Incomplete poly-Cauchy numbers", Monatsh. Math., 180: 271-288, (2016).

[11] Komatsu, T., Mezö, I. and Szalay, L., "Incomplete Cauchy numbers", Acta Math. Hungar., 149: 306-323, (2016).

[12] Komatsu, T. and Young, P.T., "Generalized Stirling numbers with poly-Bernoulli and polyCauchy numbers", Int. J. Number Theory, 14(05): 1211-1222, (2018).

[13] Komatsu, T. and Szalay, L., "Shifted poly-Cauchy numbers", Lith. Math. J., 54: 166-181, (2014).

[14] Boyadzhiev, K.N., "Polyexponentials", available from: http://arxiv.org/pdf/0710.1332v1.pdf.

[15] Rahmani, M., “On p-Cauchy numbers”, Filomat, 30(10): 2731-2742, (2016).

[16] Lah, I., "A new kind of numbers and its application in the actuarial mathematics", Bol. Inst. Actuár. Port., 9: 7-15, (1954).

[17] Rahmani, M., “Generalized Stirling transform”, Miskolc Math. Notes, 15: 677-690, (2014).

[18] Komatsu, T., "Sums of products of Cauchy numbers, including poly-Cauchy numbers", J. Discrete Math., 2013: Article ID373927, (2013).

[19] Zhao, F.Z., "Sums of products of Cauchy numbers", Discrete Mathematics, 309(12): 3830-3842, (2009).

[20] Howard, F. T., Nörlund's number $B_{n}^{n}$, Applications of Fibonacci Numbers, Vol. 5, Kluwer Acad. Publ., Dordrecht, (1993).

[21] Lacpao N., Corcino R., Vega M.A.R., "Hurwitz-Lerch type multi-poly-Cauchy numbers", Mathematics, 7(4): 335, (2019). 